\title{
Sélection des espèces indicatrices d'oiseaux des galeries forestières au Bénin (Afrique de l'Ouest)
}

\author{
Francis Biaou YABI ${ }^{1,2 *}$, Toussaint Olou LOUGBEGNON ${ }^{2}$ et \\ Jean Timothée Claude CODJIA ${ }^{2}$ \\ ${ }^{1}$ Département de Géographie et Aménagement du Territoire, Université d'Abomey-Calavi (UAC), Bénin. \\ ${ }^{2}$ Laboratoire d'Ecologie Animale et de Zoogéographie, Ecole de Foresterie et d'Ingénierie du Bois (EFIB), \\ Université d'Agriculture de Kétou (UAK), Bénin. \\ *Auteur correspondant ; E-mail : francisyabi@yahoo.fr; Tel : (+229) 95188063/97006176
}

\section{REMERCIEMENTS}

Les auteurs remercient la Fondation Internationale pour la Science (FIS) pour son appui financier pour la réalisation de cette recherche.

\section{RESUME}

Les oiseaux constituent des indicateurs biologiques de valeur pour apprécier certains types de perturbations du milieu naturel. Cette étude vise à déterminer les espèces d'oiseaux indicatrices des galeries forestières. L'observation des oiseaux a été effectuée dans les trois zones biogéographiques à savoir: zone guinéenne, zone soudano - guinéenne et la zone soudanienne au moyen d'une technique d'échantillonnage de type ponctuel axé sur les points d'écoute de 20 minutes dans 45 stations d'écoute circulaires de $18 \mathrm{~m}$ de rayon de décembre 2013 à avril 2016. Les espèces d'oiseaux indicatrices des galeries forestières dans chaque zone biogéographique ont été recherchées en utilisant l'indice de valeur indicatrice. Au total, 140 espèces d'oiseaux ont été identifiées dans les galeries forestières au Bénin. L'analyse des espèces indicatrices révèle que 89 espèces d'oiseaux sont significativement indicatrices des galeries forestières à l'échelle du Bénin. Ces analyses indiquent respectivement que 16 espèces $(11,42 \%)$ sont significativement indicatrices de la zone guinéenne, 31 $(21,14 \%)$ espèces sont significativement indicatrices de la zone soudanienne et $27(19,29 \%)$ espèces sont significativement indicatrices de la zone soudano - guinéenne. Cette recherche fournit aux décideurs des indicateurs écologiques fiables sur lesquels ils peuvent se fonder pour envisager des stratégies de conservation des habitats et des espèces d'oiseaux.

(C) 2017 International Formulae Group. All rights reserved.

Mots clés: Indicateurs écologiques, oiseaux, galeries forestières, Bénin. 


\title{
Selection of indicator bird species of the riparian forests in Benin (West Africa)
}

\begin{abstract}
Birds are valuable biological indicators for assessing certain types of natural disturbance. The aim of this study is to determine the indicator species of riparian forests. Birds were observed in the three biogeographical zones: Guinea, Sudano - Guinea and the Sudanian zone using a 20 - minute Point - of - Point sampling technique in 45 circular $18 \mathrm{~m}$ radius listening stations from December 2013 to April 2016. The indicator bird species of riparian forests in each biogeographic zone were searched using the indicator value index. A total of 140 bird species have been identified in riparian forests of Benin. Analysis of the indicator species shows that 89 bird species are significantly indicative of riparian forests in Benin. These analyse indicate respectively that 16 species $(11.42 \%)$ are significantly indicative of the Guinean zone, $31(21.14 \%)$ species are significantly indicative of the Sudanian zone and 27 (19.29\%) species are significantly indicative of the Sudano - Guinean zone. This research provides policy makers with reliable ecological indicators on which to base of strategies conservation of habitat and species.

(C) 2017 International Formulae Group. All rights reserved.
\end{abstract}

Keywords: Ecological Indicators, birds, riparian forests, Benin.

\section{INTRODUCTION}

Les espèces bio indicatrices peuvent être définie comme une espèce ou une communauté d'espèces qui est particulièrement bien adaptée à un paysage et /ou réagit aux effets des changements environnementaux (Büchs, 2003, Lougbégnon et al., 2009).

L'identification des espèces indicatrices est importante pour le suivi environnemental, à des fins d'aménagement du territoire, à la conservation et à la gestion des écosystèmes forestiers (Lougbégnon et al., 2009). Les espèces indicatrices devraient obéir à quatre règles à savoir: liées aux objectifs d'évaluation; justifier une réponse à une série de contraintes environnementales; montrer un potentiel d'intégration à long terme, être facilement quantifiable et mesurable (Carignan et Villard, 2002). Dans le contexte de bio indicateur de la santé des galeries forestières, il est indispensable aujourd'hui que pour suivre l'évolution écologique de ces milieux, il faut s'appuyer sur des bio- indicateurs fiables qui intègrent à la fois les facteurs biotiques et abiotiques du milieu. Pour ce faire, les oiseaux constituent un matériel de choix. Ce point permet de comprendre que les oiseaux constituent la meilleure résolution taxonomique pour identifier les différentes caractéristiques ou changement opérés dans les galeries forestières ( Khaffou et al., 2013; Seymour et al., 2015). Dans ce contexte de changement climatique et de modification de l'habitat, l'utilisation des espèces indicatrices devient de plus en plus nécessaire dans la gestion des systèmes écologiques (Delahaye, 2006). L'identification d'espèces ou de groupes d'espèces capables de prédire un ensemble de variables de milieu constitue la question centrale de la théorie des indicateurs biologiques (Chessel et Thioulouse, 2001). Au Bénin, les espèces végétales ont été estimées à 2807 (Akoegninou et al., 2006), 4378 espèces animales (PNUD-MEPN, 2009), et 526 espèces d'oiseaux (Lougbegnon et Libois, 2011). 
Pour ce faire, l'objectif de cette étude est de déterminer les espèces d'oiseaux indicatrices des galeries forestières de chaque zone biogéographique. Ceci est beaucoup plus importantes que ces bio indicateurs de la biodiversité peuvent guider la prise des décisions en aidant les gestionnaires à établir des priorités en leur fournissant des informations pour décrire l'objectif et déterminer si les objectifs des politiques ont été atteints (Nahmani et al., 2006). Etant donné que les gestionnaires et les décideurs sont les destinataires ultimes de ces indicateurs, il est attendu des scientifiques qu'ils mettent en évidence l'intérêt d'indicateurs spatialisés plutôt que des indicateurs stationnels, certes peut-être plus explicatifs, mais beaucoup moins mobilisables, donc efficaces (infine) pour un gestionnaire d'espace naturel.

\section{MATERIEL ET METHODES}

\section{Description du milieu d'étude}

La végétation au Bénin est à peu près divisé en 10 districts phytogéographiques (régions homogènes floristiques) regroupée en trois zones biogéographique basées sur des facteurs écologiques (climat, sol, la géologie et l'utilisation des terres) et des événements historiques (ie dernières fluctuations climatiques au cours de l'Holocène) (Adomou et al., 2006) . Les phytodistricts sont: Côté, Pobé, Vallée de l'Ouémé, du Plateau, Zou, Bassila, Sud Borgou, Nord Borgou, MékrouPendjari et Atacora Chaine (Figure 1).

L'étude a été conduite dans les galeries forestières représentant les sites d'observation des oiseaux le long du gradient bioclimatique du Bénin. Les sites ont été principalement choisis de manière à respecter le gradient bioclimatique et la variation dans la composition des galeries forestières (Figure 1). Les galeries forestières représentent après la forêt dense, le deuxième écosystème où la diversité floristique et avifaunistique est plus importante (Natta et Porembski, 2011). Elles représentent $2,37 \%$ de la couverture totale des superficies boisées au Bénin (CENATEL, 2002). Deux types de climat sont principalement distingués au Bénin: le climat subéquatorial avec deux saisons des pluies et deux saisons sèches dans la partie sud du pays et le climat tropical caractérisé par une saison sèche et une saison des pluies (Adam et Boko, 1993). Les précipitations moyennes annuelles sont d'environ 1000-1300 mm (dans le Sud), 900-1100 mm (dans la zone de transition) et 750-900 mm (dans la partie Nord). Les températures moyennes annuelles varient de 26 à $28{ }^{\circ} \mathrm{C}$ et peuvent exceptionnellement atteindre $35-40{ }^{\circ} \mathrm{C}$ dans les localités du Nord comme Kandi, Karimama et Malanville. L'amplitude annuelle de la température est faible dans la partie sud $\left(5-10{ }^{\circ} \mathrm{C}\right)$, alors qu'elle est plus élevée $\left(11-13{ }^{\circ} \mathrm{C}\right)$ dans la partie Nord (de la $8^{\circ}$ de latitude $\mathrm{N}$ vers le Nord).

\section{Matériel d'identification des oiseaux}

Le matériel d'identification des oiseaux est constitué d'une paire de jumelles pour l'observation à distance des oiseaux, d'un guide d'identification des oiseaux (Borrow et Demey, 2001) pour la reconnaissance parfaite de l'espèce à travers son plumage et nom scientifique, des filets japonais pour la capture des oiseaux, d'un appareil photographique pour la prise des vues des oiseaux non identifiés ou d'identification douteuse sur le terrain et d'un GPS (Global Positioning System) pour la prise des coordonnées des placeaux, des cartes topographiques, une pirogue, des fiches de 
relevés. La nomenclature des oiseaux est celle utilisée dans l'ouvrage de Borrow et Demey (2001).

\section{Méthodes de collectes des données Echantillonnage}

Pour conduire cette étude, les galeries forestières suivant les trois zones biogéographiques du Bénin ont été considérées: les galeries forestières de la zone soudanienne, les galeries forestières de la zone de soudano-guinéenne et les galeries forestières de la zone guinéenne. A l'échelle du Bénin, neuf sites d'observation ont été choisis à raison de trois sites d'observation par zone climatique. Les sites d'observations des oiseaux ont été choisis en tenant compte des modes d'occupations des terres allant des Parcs (Parc W et le Parc Pendjari) en passant par les forêts classées et enfin les zones non protégées (Tableau 1). Ainsi, un total de 15 placeaux est posé dans chaque zone climatique à raison de 5 placeaux par sites d'observations, ce qui fait 45 placeaux sur tout l'étendu de territoire national (Tableau 2).

Méthode de recensement de l'avifaune des galeries forestières

L'observation des oiseaux est faite à travers des points d'écoute d'oiseau placé au centre de chaque placeau. Les points d'écoute sont effectués suivant la technique d'échantillonnages stratifiés de types ponctuels (EPS) précédés d'une mémorisation des chants des oiseaux. La durée d'un point d'écoute est de 20 minutes. La capture des oiseaux s'est faite au moyen du filet japonais. Le filet japonais est utilisé afin de capturer les oiseaux des sous-bois et ceux qui échapperont à la vue. Compte tenu de l'aspect très exigu et fermé des galeries forestières, le rayon de détection des oiseaux est limité à un cercle de $18 \mathrm{~m}$. Au-delà, la détection de l'oiseau n'est plus prise en compte.

Pour chaque station, la richesse spécifique et l'abondance des espèces aviennes ont été prises en compte. Ainsi, dans chaque station, toutes les espèces d'oiseaux vues ou entendues sont enregistrées.

L'étude s'est déroulée de 2013 à 2016. Chaque station est prospectée trois fois par jours entre $6 \mathrm{~h}$ et $18 \mathrm{~h} 30 \mathrm{mn}$ mais les jours d'orage où de fortes pluies sont évités.

\section{Description des stations d'écoute et d'observation des oiseaux}

La description des stations d'écoute des oiseaux est consignée dans le Tableau 2. De l'analyse de ce tableau, il ressort que les trois zones biogéographiques (zone Guinéenne, zone Soudano-guinnéenne et zone Soudanienne) ne présentent pas les mêmes caractéristiques écologiques. La zone Guinéenne prend en compte les S1 à S15. Elle est caractérisée par des arbres de hauteur variant généralement entre 8 et $24 \mathrm{~m}$. Le recouvrement au sol de la végétation est estimé à $85 \%$. La strate herbacée est aussi présente. On note dans ces stations la présence d'activité anthropique. Ces galeries forestières sont plus concentrées hors des zones protégées. La zone Soudano - guinéenne s'étend de la station S16 à S30. Cette zone est dominée par les arbres dont les hauteurs varient entre 8 et $20 \mathrm{~m}$. Le recouvrement au sol de la végétation tourne autour de $35 \%$. La strate herbacée domine beaucoup plus. On remarque une intense activité anthropique due à la coupe des espèces végétales ligneuses et l'activité de la carbonisation. Enfin, la zone Soudanienne prend en compte la station S31 à S45. Le descripteur écologique est l'absence quasi-totale des activités humaines. On note aussi l'isolement de quelques espèces ligneuses réparties en faible proportion. Ces stations sont à cheval entre deux parcs (Parcs W et le Parc Pendjari) et une forêt classée (Forêt classée de la Sota).

\section{Méthode de recherches des espèces indicatrices}

Les espèces indicatrices de chaque zone biogéographique ont été identifiées en 
utilisant la méthode statistique des espèces indicatrices grâces au logiciel IndVal (Dufrêne, 2001). Le test de permutation Monte carlo incorporé dans cette méthode a permi d'identifier les espèces indicatrices. La statistique du test (IndVal) combine la fidélité des espèces (proportions de station d'un groupe où l'espèce est présente) et leur spécificité (à quel point une espèce ne se trouve que dans le groupe considéré). Une espèce est considérée comme indicatrice si elle est typique d'une classe d'habitat ou d'un groupe de classe et si elle est présente dans tous les relevés de cette classe d'habitat ou de ce groupe de classes. La spécificité est maximale quand l'espèce n'occupe qu'une classe ou qu'un groupe et la fidélité est maximale lorsque l'espèce est présente dans tous les relevés d'une classe ou d'un groupe de classes. Ainsi, la présence/absence des espèces selon les classes d'habitats définies (galeries forestières de la zone soudanienne, galeries forestières de la zone soudano guinéenne et les galeries forestières de la zone guinéenne) a été analysée par cette méthode. Les espèces qui sont significativement associées à un type d'habitat donné/ groupe d'habitats, dont la valeur IndVal est maximale en considérant toutes les partitions étudiées sont recherchées.

\section{Analyses statistiques des données avifaunistiques}

Une matrice de données de présence/absence des oiseaux des galeries forestière a été utilisée pour la détermination des espèces d'oiseaux indicateurs de chaque zone climatique. Pour chaque communauté d'oiseaux et chaque zone climatique, les espèces indicatrices ont été sélectionnées numériquement suivant la méthode de Dufrêne et Legendre (1997). La détermination des espèces indicatrices dans chaque zone biogéographique a été faite en ajoutant à la matrice de données initiales de relevés une variable supplémentaire décrivant le type groupement dans lequel l'ensemble des stations appartenaient. La même règle a été utilisée pour déterminer les espèces d'oiseaux indicatrices dans la communauté. Le module « Indicators species Analysis» basé dans le logiciel PC-Ord a été utilisé pour déterminer la valeur Indicatrice (VI) de chaque espèce dans chaque zone biogéographique. La valeur Indicatrice est la combinaison de l'abondance relative de chaque espèce d'oiseau (Ar en\%) et la fréquence relative ( $\mathrm{Fr}$ en\%) dans chaque zone climatique.

L'abondance relative des oiseaux a été donnée par la formule :

$$
A_{r}=\frac{n_{i}}{N}
$$

Avec ni le nombre d'individu de l'espèce i dans le groupement et $\mathrm{N}$ le total le nombre total des individus des espèces du groupe. L'abondance relative mesure la spécificité de l'espèce i dans le groupement.

La fréquence relative $F_{r}$ a été donnée par la formule :

$$
F_{r}=\frac{n_{s i}}{N}
$$

Avec $n_{\text {si }}$ le nombre de station dans lequel l'espèce i se retrouve dans le groupement et $\mathrm{N}$ le nombre total de station dans le groupement. La fréquence relative quant à elle mesure donc la fidélité de l'espèce dans le groupement. En définitive, la valeur indicatrice est donnée par la formule:

$$
V I=A r \times F r \times 100
$$

Le test de permutation de Monte Carlos a été effectué sur les valeurs Indicatrices pour déterminer les espèces d'oiseaux dont la valeur Indicatrice est significative. Une espèce est indicatrice d'un milieu si et seulement si elle a une Valeur Indicatrice inférieure ou égale à $25 \%$ et le test de Monte Carlos est significatif au seuil $(\mathrm{P}<0,05)$ (Dufrêne et Legendre 1997, Nahmani et al., 2006, Lougbégnon et al., 2009). 


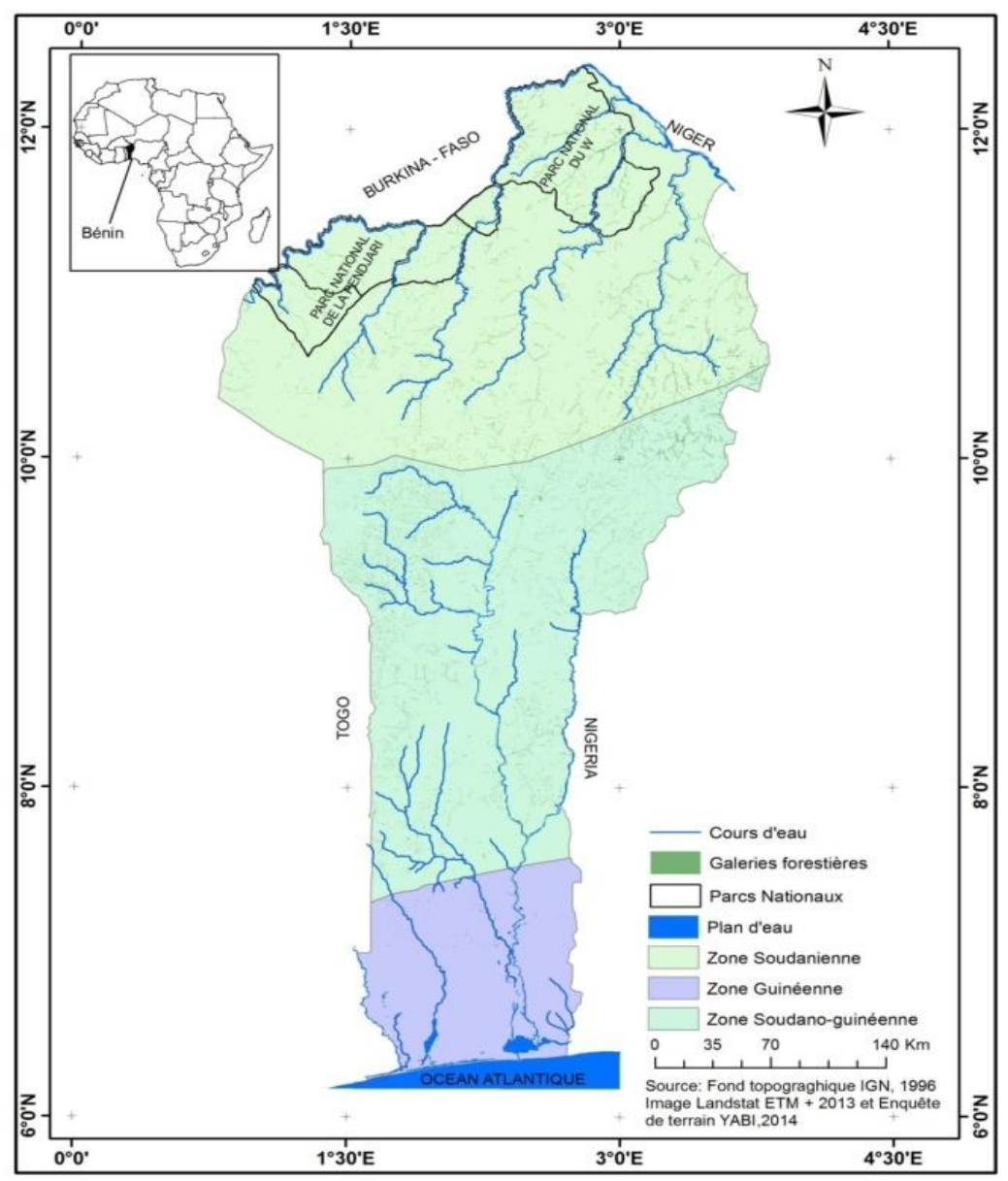

Figure 1: Localisation du milieu d'étude.

Tableau 1: Récapitulatif des sites d'échantillonnage par zone climatiques.

\begin{tabular}{lccc}
\hline Zones biogéographiques & $\begin{array}{c}\text { Nombre de } \\
\text { placeaux }\end{array}$ & Secteurs & Cours d'eau \\
\hline Guinéenne & 5 & Zone non protégée & Fleuve Couffo \\
& 5 & Zone non protégée & Fleuve Ouémé \\
& 5 & Zone non protégée & Fleuve Mono \\
\hline Soudano - guinéenne & 5 & Zone non protégée & Rivière Okpara \\
& 5 & Forêts classées des Monts & Fleuves Ouémé \\
& 5 & Fouffé & \\
& & Fôt classée de l'ouémé & Rivières Affon \\
& 5 & Parc Pendjari & \\
\hline Soudanienne & 5 & Parc W & Rivières Pendjari \\
& 5 & Forêt classée de la Sota & Rivière Sota \\
\hline
\end{tabular}


Tableau 2 : Description des stations d'écoute des oiseaux.

\begin{tabular}{|c|c|c|c|}
\hline Stations d'écoute & Latitudes & Longitudes & Description des stations \\
\hline S1 & 7,31846 & 1,75582 & $\begin{array}{l}\text { Cette station est située sur le fleuve Couffo. La } \\
\text { hauteur des arbres oscille entre } 8 \mathrm{~m} \text { et } 24 \mathrm{~m} \text {. Le } \\
\text { recouvrement de la végétation au sol est estimé à } \\
55 \% \text {. La largeur de la bande verte est à l'ordre } 5 \mathrm{~m} \text {. }\end{array}$ \\
\hline S2 & 7,26606 & 1,80525 & $\begin{array}{l}\text { De même que la station S1, S2 est aussi située sur le } \\
\text { fleuve Couffo à l'est de rive. Elle est composée des } \\
\text { arbres dont les hauteurs tournent autour de } 5 \mathrm{~m} \text { et } \\
20 \mathrm{~m} \text {. le recouvrement de la végétation au sol est à } \\
\text { l'ordre de } 70 \% \text {. La largeur de la partie occupée par } \\
\text { la végétation est de } 7 \mathrm{~m} \text { environ. }\end{array}$ \\
\hline $\mathbf{S 3}$ & 7,20047 & 1,84377 & $\begin{array}{l}\text { Cette station s'ouvre sur le fleuve Couffo. C'est une } \\
\text { galerie à Alchornea cordifolia avec un recouvrement } \\
\text { total au sol évalué à } 45 \% \text {. La largeur de la galerie est } \\
4,5 \mathrm{~m} \text {. }\end{array}$ \\
\hline S4 & 7,13832 & 1,86285 & $\begin{array}{l}\text { Elle est localisée sur le fleuve Couffo. C'est une } \\
\text { galerie forestière à Ficus congensis. Cette station } \\
\text { comporte } 90 \% \text { d'espèces herbacées typiques des } \\
\text { zones humides au Sud du Bénin. Le recouvrement } \\
\text { moyen végétal est de } 90 \% \text { herbacée et } 30 \% \text { arborée. } \\
\text { La largeur de la galerie forestière est de } 5 \mathrm{~m} \text {. }\end{array}$ \\
\hline S5 & 7,08783 & 1,88798 & $\begin{array}{l}\text { Cette station se situe sur le fleuve couffo près d'une } \\
\text { grande plantation de l'espèce Elaeis guineensis. En } \\
\text { dehors du lit majeur du cours d'eau qui constitue } \\
\text { environ } 10 \% \text {, il y a une surface considérable }(70 \%) \\
\text { dont la couverture végétale est essentiellement } \\
\text { ligneuse (Raphia hookeri, Nauclea zanthoxylon). }\end{array}$ \\
\hline S6 & 6,35295 & 1,79124 & $\begin{array}{l}\text { C'est une station qui fait corps au lit majeur du } \\
\text { fleuve Mono. La végétation est constituée des } \\
\text { espèces végétales Elaeis guineensis couvrant le sol à } \\
60 \% \text {. }\end{array}$ \\
\hline S7 & 6,41926 & 1,77749 & $\begin{array}{l}\text { Elle est aussi située sur le fleuve Mono. Le } \\
\text { descripteur écologique de cette station est la } \\
\text { dominance des activités agricoles. La largeur de } \\
\text { cette galerie forestière tourne autour de } 4 \mathrm{~m} \text {. }\end{array}$ \\
\hline $\mathbf{S 8}$ & 6,47010 & 1,74610 & $\begin{array}{l}\text { Elle est localisée sur le fleuve Mono. La hauteur de } \\
\text { la végétation varie entre } 8 \text { et } 20 \mathrm{~m} \text {. Le recouvrement } \\
\text { au sol de la végétation tourne autour de } 35 \% \text {. La } \\
\text { strate herbacée domine beaucoup plus. }\end{array}$ \\
\hline S9 & 6,49426 & 1,71387 & $\begin{array}{l}\text { Elle est installée sur le fleuve Mono. La hauteur des } \\
\text { arbres varie généralement entre } 8 \text { et } 24 \mathrm{~m} \text {. Le } \\
\text { recouvrement au sol de la végétation est estimé à } \\
85 \% \text {. La strate herbacée est aussi présente. }\end{array}$ \\
\hline S10 & 6,53890 & 1,70050 & $\begin{array}{l}\text { Cette station est située sur le fleuve Mono. La } \\
\text { proportion des espaces couverts par la végétation } \\
\text { occupe } 20 \% \text {. Les arbres caractéristiques de cette }\end{array}$ \\
\hline
\end{tabular}




\begin{tabular}{|c|c|c|c|}
\hline & & & 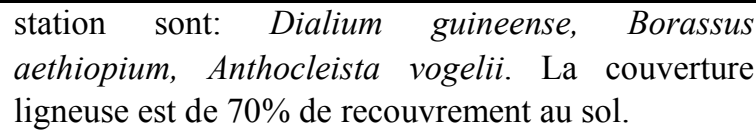 \\
\hline S11 & 7,31634 & 2,43383 & $\begin{array}{l}\text { C'est une station localisée sur le fleuve Ouémé dans } \\
\text { la grande vallée de l'Ouémé, un milieu très humide. } \\
\text { Elle couvre à la fois une partie boisée et une autre de } \\
\text { frange d'eau libre. La végétation de la partie boisée } \\
\text { est faite Drypetes floribunda, Pterocarpus } \\
\text { santalinoides , Parinari congensis à } 70 \% \text {. La } \\
\text { largeur de la galerie forestière est estimée à environ } \\
10 \mathrm{~m} \text {. }\end{array}$ \\
\hline S12 & 7,23491 & 2,45086 & $\begin{array}{l}\text { Cette station est située sur le fleuve Ouémé. La } \\
\text { hauteur de la végétation oscille entre } 12 \text { et } 24 \mathrm{~m} \\
\text { tandis que le degré de recouvrement est estimé } 90 \% \text {. }\end{array}$ \\
\hline S13 & 7,19842 & 2,44728 & $\begin{array}{l}\text { Cette station d'échantillonnage se trouve sur le } \\
\text { fleuve Ouémé. Elle est parsemée par des espèces } \\
\text { comme Pterocarpus santalinoides, Albizyia } \\
\text { glaberrima, Sterculia dont la hauteur varie entre } 10 \\
\text { et } 25 \mathrm{~m} \text {. La couverture végétale est de l'ordre de } \\
80 \% \text {. }\end{array}$ \\
\hline S14 & 7,36399 & 2,38427 & $\begin{array}{l}\text { C'est une station située dans une zone bien } \\
\text { aménagée et entretenue par l'homme qui se situe sur } \\
\text { le fleuve Ouémé. Les arbres fruitiers atteignent une } \\
\text { hauteur de } 16 \mathrm{~m} \text {. La proportion des franges d'eau } \\
\text { libre est estimée à } 10 \% \text {. }\end{array}$ \\
\hline S15 & 7,10239 & 2,42151 & $\begin{array}{l}\text { C'est une station située sur le fleuve Ouémé. C'est } \\
\text { une galerie forestière à Cynometra megalophylla, } \\
\text { Trilipiseum madagascariense, Cleistopholis patens } \\
\text { avec un recouvrement de } 90 \% \text { au sol. Les franges } \\
\text { d'eau libre occupent petite partie de la station (15\%). }\end{array}$ \\
\hline S16 & 9,63770 & 2,36450 & $\begin{array}{l}\text { Elle est localisée sur la rivière Okpara dans la zone } \\
\text { Soudano -guinéenne. Elle couvre une partie de } \\
\text { frange d'eau libre ( } 20 \% \text { ) et une partie semi } \\
\text { inondable composée de quelques pieds de Berlinia } \\
\text { grandifolia, Elaeis guineensis, Pouteria alnifolia de } \\
\text { hauteur variante entre } 10 \text { et } 18 \mathrm{~m} \text { avec un taux de } \\
\text { recouvrement de } 75 \% \text { au sol. }\end{array}$ \\
\hline S17 & 9,59439 & 2,35154 & $\begin{array}{l}\text { Elle est localisée sur la rivière Okpara. Elle s'ouvre } \\
\text { directement à la rivière. C'est une station qui } \\
\text { présente les mêmes caractéristiques que la } \\
\text { précédente. }\end{array}$ \\
\hline S18 & 9,53184 & 2,32046 & $\begin{array}{l}\text { C'est une station située sur la rivière Okpara à } \\
\text { proximité de la voie reliant le village Oké owo au } \\
\text { Nigéria. La végétation ici est composée de } \\
\text { Lecaniodiscus cupanoides, Napoleonea vogelii et } \\
\text { Uvaria chamae avec un recouvrement au sol } 60 \% \text {. }\end{array}$ \\
\hline S19 & 9,42405 & 2,29994 & $\begin{array}{l}\text { Elle est aussi localisée sur la rivière Okpara. Elle est } \\
\text { caractérisée par une strate arborescente allant jusqu'à }\end{array}$ \\
\hline
\end{tabular}




\begin{tabular}{|c|c|c|c|}
\hline & & & $\begin{array}{l}20 \mathrm{~m} \text { et dominée par Hexalobus crispiflorus, } \\
\text { Pouteria alnifolia avec un recouvrement de } 30 \% \text {; } \\
\text { une strate herbacée de } 1 \text { à } 2 \mathrm{~m} \text { et dominée par Typha } \\
\text { australis, Alchorrnea cordifolia, et le taux de } \\
\text { recouvrement est estimé à } 85 \% \text {. }\end{array}$ \\
\hline S20 & 9,36296 & 2,29222 & $\begin{array}{l}\text { C'est un milieu humide situé sur la rivière Okpara. } \\
\text { Le couvert végétal au sol est occupé par un tapis } \\
\text { herbacé. On note également la présence d'une } \\
\text { végétation ligneuse telle que Berlinia grandifolia, } \\
\text { Elaeis guineensis, Uvaria chamae de degré de } \\
\text { recouvrement } 70 \% \text {. Le sol est du type argilo- } \\
\text { sableux. }\end{array}$ \\
\hline S21 & 8,78793 & 2,19420 & $\begin{array}{l}\text { Cette station est située sur le fleuve Ouémé dans la } \\
\text { région de Idadjo et est située dans la forêt classée des } \\
\text { Monts Kouffé. La surface est occupée presque } \\
\text { exclusivement d'eau et de végétation (Dialium } \\
\text { guineense, Anogeissus leiocarpus, Isoberlinia } \\
\text { tomentosa, Isoberliia doka). La hauteur de la } \\
\text { végétation varie entre } 10 \text { à } 20 \mathrm{~m} \text { avec un taux de } \\
\text { recouvrement au sol de } 85 \% \text {. }\end{array}$ \\
\hline S22 & 8,71812 & 2,20550 & $\begin{array}{l}\text { Cette station est localisée dans la forêt classée des } \\
\text { Monts Kouffé sur le fleuve Ouémé. Elle présente les } \\
\text { caractéristiques ci-après: une strate arborée de } 8 \text { à } 20 \\
\mathrm{~m} \text { dominée par Diospyros mespilliformis, Isoberlinia } \\
\text { doka, Dialium guineense, d'un taux de recouvrement } \\
\text { de } 70 \% \text {. }\end{array}$ \\
\hline $\mathbf{S 2 3}$ & 8,60282 & 2,22108 & $\begin{array}{l}\text { Cette station se situe sur le fleuve Ouémé dans la } \\
\text { forêt classée des Monts kouffé. En dehors de } \\
\text { l'espace boisé qui constitue environ } 70 \% \text {, il y a une } \\
\text { surface considérable constitué de frange d'eau libre } \\
\text { avec une proportion de } 30 \% \text { dont la couverture } \\
\text { végétale est essentiellement herbacée. }\end{array}$ \\
\hline S24 & 8,56156 & 2,21273 & $\begin{array}{l}\text { C'est une station située dans la forêt classée des } \\
\text { Monts Kouffé. Le sous-bois est dense avec un taux } \\
\text { de recouvrement de } 90 \% \text { et } 10 \% \text { d'arbre. }\end{array}$ \\
\hline S25 & 8,51092 & 2,23477 & $\begin{array}{l}\text { C'est une galerie forestière à Pterocarpus } \\
\text { santalinoides, Cynometra megalophylla, avec une } \\
\text { hauteur qui varie entre } 7 \text { et } 18 \mathrm{~m} \text { et d'un taux de } \\
\text { recouvrement de } 10 \% \text {. Le sous-bois est dense avec } \\
\text { un taux de recouvrement } 90 \% \text {. }\end{array}$ \\
\hline S26 & 7,73361 & 2,54008 & $\begin{array}{l}\text { Cette station est située également sur le fleuve } \\
\text { Ouémé. La surface échantillonnée est occupée par } \\
\text { une frange d'eau libre, un espace boisé avec des } \\
\text { espèces végétales comme Pterocarpus santaloides, } \\
\text { Cola laurifolia, syzygium guineense. }\end{array}$ \\
\hline S27 & 7,82401 & 2,60003 & $\begin{array}{l}\text { Elle est située sur la rivière Affon dans la forêt } \\
\text { classée de l'Ouémé supérieure dans la zone soudano } \\
\text { - guinéenne. Cette station une galerie forestière } a\end{array}$ \\
\hline
\end{tabular}




\begin{tabular}{|c|c|c|c|}
\hline & & & $\begin{array}{l}\text { Berlinia grandifolia et jalonnée Syzygium guineense } \\
\text { avec un recouvrement au sol de } 50 \% \text {. De même elle } \\
\text { est traversée par une frange d'eau libre occupant } \\
20 \% \text { de recouvrement au sol de la station. }\end{array}$ \\
\hline S28 & 7,76121 & 2,57469 & $\begin{array}{l}\text { Elle est située sur la rivière Affon dans la forêt } \\
\text { classée de l'Ouémé supérieure. Cette station est une } \\
\text { galerie forestière à Xylopia parviflora et Diospyros } \\
\text { mespiliformis jalonnée de quelques pieds de Elaeis } \\
\text { guineensis et de Borassus aethiopum avec un } \\
\text { recouvrement de } 50 \% \text {. De même, elle est parsemée } \\
\text { par une frange d'eau libre occupant } 20 \% \text { de } \\
\text { recouvrement total de la station. }\end{array}$ \\
\hline S29 & 7,84343 & 2,64511 & $\begin{array}{l}\text { Cette station est localisée dans la rivière Affon dans } \\
\text { la zone soudano - guinéenne. Elle est composée } \\
\text { d'un sous-bois fermé et présente les caractéristiques } \\
\text { ci-après: une strate Elaeis guineenesis, Mitragina } \\
\text { inermis de hauteur } 10 \mathrm{~m} \text { environs dont le taux de } \\
\text { recouvrement est estimé à } 25 \% \text {; un sous-bois de } \\
\text { Fridelia ferruginea de Anona senegalensis d'un taux } \\
\text { de recouvrement de } 75 \% \text { environ. Il faut signaler } \\
\text { aussi qu'à l'intérieur de cette galerie forestière, on } \\
\text { note la présence des franges d'eau libre. }\end{array}$ \\
\hline S30 & 7,89781 & 2,65567 & $\begin{array}{l}\text { Elle est située à la lisière de la forêt classée de la } \\
\text { Sota et se caractérise par : un espace boisé de } 80 \% \\
\text { de recouvrement; une végétation constituée de } \\
\text { quelques pieds de Diospyros mespiliformis, Parinari } \\
\text { congensis. A proximité de cette station se trouve la } \\
\text { forêt classée de l'Ouémé supérieure. }\end{array}$ \\
\hline S31 & 11,13934 & 3,23983 & $\begin{array}{l}\text { C'est une galerie forestière située sur la rivière Sota } \\
\text { et possède des caractéristiques similaires à la station } \\
\text { 30. On y note aussi la présence de végétations faites } \\
\text { de Manikara multinervis et de Pterocarpus } \\
\text { santalinoides. }\end{array}$ \\
\hline S32 & 11,10460 & 3,23279 & $\begin{array}{l}\text { Elle est localisée dans la forêt classée de la Sota. Les } \\
\text { franges d'eau libre occupent une bonne partie de la } \\
\text { station et la petite surface restante est composée de } \\
\text { quelques pieds Drypetes floribunda de hauteur } \\
\text { variant entre } 10 \text { et } 15 \mathrm{~m} \text { et d'un taux de } \\
\text { recouvrement estimé à } 5 \% \text {; }\end{array}$ \\
\hline S33 & 11,06261 & 3,24365 & $\begin{array}{l}\text { Elle est localisée sur la rivière Sota dans la zone } \\
\text { soudanienne et se caractérise par la végétation } \\
\text { ligneuse quasiment inexistante composée de } \\
\text { quelques pieds Raphia sudanica, Uapaca togoensis à } \\
5 \% \text { de recouvrement. Le sol est couvert en partie } \\
\text { d'herbacée à } 50 \% \text {; }\end{array}$ \\
\hline S34 & 11,02947 & 3,24452 & $\begin{array}{l}\text { Elle est située sur le continuum que la station } \\
\text { précédente donc elle présente la même physionomie } \\
\text { et même caractéristique. }\end{array}$ \\
\hline
\end{tabular}




\begin{tabular}{|c|c|c|c|}
\hline $\mathbf{S 3 5}$ & 10,98490 & 3,25490 & $\begin{array}{l}\text { Cette station est localisée sur la rivière Sota. Une } \\
\text { partie de cette station est située sur la terre ferme et } \\
\text { une moitié est faite de frange d'eau libre dépourve } \\
\text { de végétation. Elle est caractérisée par les espèces } \\
\text { végétales comme Khaya senegalensis, Parinari } \\
\text { congensis d'un taux de recouvrement à l'ordre de } \\
65 \% \text {. }\end{array}$ \\
\hline S36 & 11,66489 & 2,93410 & $\begin{array}{l}\text { C'est une station située sur la rivière Alibori à } \\
\text { l'intérieure du Parc } \mathrm{W} \text { au Bénin. Cette station fait } \\
\text { corps à une savane arbustive. La hauteur des arbres } \\
\text { composant cette station varie généralement entre } 6 \text { et } \\
18 \mathrm{~m} \text {. Le recouvrement au sol de la végétation est } \\
\text { estimé à } 55 \% \text {. La strate herbacée est aussi présente. }\end{array}$ \\
\hline S37 & 11,65320 & 2,89841 & $\begin{array}{l}\text { Cette station se situe sur le même continuum que } \\
\text { celle de } 36 \text {. A la seule différence que celle-ci est } \\
\text { jonchée d'affleurement rocheux sur le lit majeur du } \\
\text { cours d'eau et conserve toute l'année l'eau. }\end{array}$ \\
\hline S38 & 11,74671 & 2,97590 & $\begin{array}{l}\text { C'est une station située dans le parc W. Cette station } \\
\text { est une galerie forestière à Cola laurifolia, Syzygium } \\
\text { guineense, Khaya senegalensis et Diospyro } \\
\text { mespiliformis. La hauteur de la végétation varie de } 5 \\
\text { à } 20 \mathrm{~m} \text { et le recouvrement des espèces végétales au } \\
\text { sol est de } 70 \% \text {. }\end{array}$ \\
\hline S39 & 11,79621 & 3,00811 & $\begin{array}{l}\text { Station située sur la rivière Alibori dans le parc } \mathrm{W} \\
\text { dans les terroirs riverains de la commune de } \\
\text { Karimama. Elle est une galerie forestière à Craveta } \\
\text { adansonii, Morelia senegalensis, Scleroclarya } \\
\text { birrea de hauteur variant entre } 10 \text { et } 17 \mathrm{~m} \text { avec un } \\
\text { recouvrement au sol de } 85 \% \text {. }\end{array}$ \\
\hline S40 & 11,82245 & 3,00801 & $\begin{array}{l}\text { C'est une station située non loin du Pond Pendjari } \\
\text { sur la rivière Pendjari. C'est une galerie forestière } \\
\text { caractérisée par Mitragyna inermis, Ficus spp et } \\
\text { Sacocephalus latifolius. La hauteur de la végétation } \\
\text { varie de } 8 \text { à } 18 \mathrm{~m} \text {. Le taux de recouvrement est de } \\
75 \% \text {. }\end{array}$ \\
\hline S41 & 11,11818 & 1,74707 & $\begin{array}{l}\text { Cette station est située sur la rivière Pendjari à } \\
\text { l'intérieure de la Réserve de Biosphères } \\
\text { transfrontalière de la Pendjari. La Hauteur de la } \\
\text { végétation varie entre } 8 \text { et } 20 \mathrm{~m} \text {. Le recouvrement au } \\
\text { sol de la végétation tourne autour de } 35 \% \text {. La strate } \\
\text { herbacée domine beaucoup plus. }\end{array}$ \\
\hline S42 & 11,25247 & 1,82943 & $\begin{array}{l}\text { C'est une station située dans le parc Pendjari au } \\
\text { Bénin. Cette station est une galerie forestière à Celtis } \\
\text { integrifolia, Kigelia africana, et Vitex doniana. La } \\
\text { hauteur de la végétation varie de } 10 \text { à } 20 \mathrm{~m} \text { et le } \\
\text { recouvrement des espèces végétales au sol est de } \\
85 \% \text {. }\end{array}$ \\
\hline $\mathbf{S 4 3}$ & 11,29666 & 1,86952 & Elle est située sur le continuum que la station \\
\hline
\end{tabular}




\begin{tabular}{|c|c|c|c|}
\hline & & & $\begin{array}{l}\text { précédente donc elle présente la même physionomie } \\
\text { et même caractéristique. }\end{array}$ \\
\hline S44 & 11,34622 & 1,89436 & $\begin{array}{l}\text { Galerie forestière située le long de la rivière } \\
\text { Pendjari. Elle est caractérisée par les espèces } \\
\text { végétales comme Diospyros mespiliformis, Syzygium } \\
\text { guineense, Khaya senegalensis. La hauteur de la } \\
\text { végétation varie de } 10 \text { à } 25 \mathrm{~m} \text { et le de recouvrement } \\
\text { est estimé à environ } 80 \% \text { de la superficie totale de } \\
\text { station. }\end{array}$ \\
\hline $\mathbf{S 4 5}$ & 11,40090 & 1,91915 & $\begin{array}{l}\text { Cette station est située également sur la rivière } \\
\text { Pendjari ayant les mêmes caractéristiques } \\
\text { écologiques à la seule différence de la présence des } \\
\text { affleurements rocheux sur celle-ci. }\end{array}$ \\
\hline
\end{tabular}

\section{RESULTATS \\ Espèces indicatrices suivant les zones climatiques et le degré de conservation des galeries forestières}

$\mathrm{Au}$ total, 140 espèces d'oiseaux réparties dans 18 ordres et 44 familles ont été recensées dans les forêts galeries à travers les trois zones climatiques en présence au Bénin. La richesse spécifique est plus élevée ( 72 espèces) dans la zone Guinéenne suivie de la zone Soudanienne avec (70 espèces; la zone Soudano -guinéenne a enregistré la plus faible richesse spécifique en espèces d'oiseaux. Les calculs des indices de valeurs indicatrices pour les trois zones biogéographiques indiquent respectivement 16 espèces $(11,42 \%)$ significativement indicatrices de la zone guinéenne, $31 \quad(21,14 \%)$ espèces significativement indicatrices de la zone soudanienne et $27 \quad(19,29 \%)$ espèces significativement indicatrices de la zone soudano - guinéenne. Le Tableau 3 fait état des espèces d'oiseaux indicatrices, leurs valeurs indicatrices et la probabilité associée par zone bioclimatique.

L'analyse avifaunistique basée sur les valeurs indicatrices des espèces d'oiseaux dans chaque zone climatique a relevé que les espèces d'oiseaux: Batis poentis, Bleda canicapillus, Chlorocichla Simplex, Cyanomitra olivaceaus, Illadopsis fulvescens,
Indicator maculatus, Lybius bidentatus, Merops pusillus, Nicator chloris, Pachycoccyx audeberti, Thescelocichla leucopleura, Macronyx croceus, Nigrita bicolor, Ploceus nigricollis, Terpsiphone rufiventer sont des espèces ayant des valeurs indicatrices élevées variant de 23,5 à 41,2\% avec une probabilité associée hautement significative $(P<0,05)$. Ce qui permet de déclarer ces communautés d'oiseaux indicatrices de la zone Guinéenne. Cette zone est fortement menacée par des activités anthropiques, ce qui a fortement contribué au morcellement des galeries forestières. De même, on note une absence totale des aires protégées dans cette zone pourtant qui regorge une faune avienne spectaculaire. Les espèces d'oiseaux, Ardeola raloides et Vidua Amadina fasciata, Anaplectes rubriceps, Anastomus lamelligerus, Anhinga rufa, Anthoscopus parvulus, Ciconia abdimii, Ciconia episcopus, Ciconia nigra, Cinnyris pulchellus, Circus pygargus, Cossypha albicapilla, Elminia longicauda, Ephippirrhynchus senegalensis, Estrilida caerulescens, Estrilida tryglodytes, Euodice cantans, Laniarus barbarus, Merops bulocki, Merops nubicus, Melaenormis edoloides, Mycteria ibis, Oriolus auratus, Plegadis falcinellus, Ploceus vitellinus, Pluvianus aegyptius, Rhinoptilus chalcopterus, Podica senegalensis, 
Ptilopachus petrosus, Tockus nasitus ont une valeur indicatrice comprise entre 23,1 à $46,2 \%$ et les valeurs de probabilités de chaque espèces associées à ces valeurs sont hautement significatives $(\mathrm{P}<0,05)$. Ce qui démontre que ces espèces sont indicatrices de la zone soudanienne. La photo $1 \mathrm{~A}$ et $\mathrm{B}$ présente respectivement Merops bulocki et Cossypha albicapilla capturés dans les galeries forestières de la zone soudanienne.

Les espèces d'oiseaux, Anthreptes longuemarei, Apalis flavida, Campephaga phoenicea, Glaucydium perlatum, Gypohierax angolensis, Lagonosticta larvata, Merops hirundineus, Permis apivorus, Ploceus melanocephalus, Tockus fasciatus, Vanellus spinosus, Vidua chalybeata, Ardea melanocephala, Lybius dubius, Musophaga violacea, Numida meleagris ont une valeur indicatrice oscillante entre 20 et $53,3 \%$ avec des valeurs de probabilités associées inférieures ou égales à $0,05(\mathrm{P} \leq 0,05)$. Ce qui démontre statistiquement que ces espèces d'oiseaux sont indicatrices des galeries forestières de la zone soudano - guinéenne. La Photo 2 A et B présente Musophaga violacea et Apalis flavida contactés dans les galeries forestières de la zone soudano - guinéenne.

Tableau 3 : Valeurs indicatrices des espèces d'oiseaux dans chaque zone climatique.

\begin{tabular}{|c|c|c|c|}
\hline Zones biogéographiques & Espèces d'oiseaux & IV & P_values \\
\hline \multirow[t]{16}{*}{ Guinéenne } & Accipiter badius & 66,7 & 0,0002 \\
\hline & Alethe diademata & 66,7 & 0,0002 \\
\hline & Andropadus Virens & 80 & 0,0002 \\
\hline & Andropadus curvirostris & 73,3 & 0,0002 \\
\hline & Andropadus gracilirostris & 46,7 & 0,0008 \\
\hline & Ardea purpurea & 40 & 0,0024 \\
\hline & Batis poensis & 86,7 & 0,0002 \\
\hline & Bleda canicapillus & 46,7 & 0,0008 \\
\hline & Centropus grillii & 33,3 & 0,0106 \\
\hline & Cyanomitra olivaceus & 40 & 0,0014 \\
\hline & Lybius bidentatus & 40 & 0,0012 \\
\hline & Merops pusillus & 33,3 & 0,0072 \\
\hline & Adeola raloides & 13,3 & 0,7091 \\
\hline & Amaurornis flavorista & 10 & 0,8528 \\
\hline & Tauraco persa & 10 & 0,858 \\
\hline & Vidua macroura & 12 & 0,5873 \\
\hline \multirow[t]{12}{*}{ Soudano- guinéenne } & Accipiter tachiro & 12 & 0,5999 \\
\hline & Ardea cinerea & 10 & 0,8536 \\
\hline & Bycanites fistulator & 12 & 0,5857 \\
\hline & Anthreptes longuemarei & 93,3 & 0,0002 \\
\hline & Apalis flavida & 66,7 & 0,0002 \\
\hline & Campephaga phoenicea & 53,3 & 0,0002 \\
\hline & Egretta alba & 66,7 & 0,0002 \\
\hline & Glaucydium perlatum & 66,7 & 0,0002 \\
\hline & Guttera pucherani & 53,3 & 0,0002 \\
\hline & Gypohierax angolensis & 26,7 & 0,0306 \\
\hline & Halcyon leucocephala & 53,3 & 0,0002 \\
\hline & Halcyon senegalensis & 73,3 & 0,0002 \\
\hline
\end{tabular}




\begin{tabular}{|c|c|c|c|}
\hline & Lagonosticta larvata & 33,3 & 0,0074 \\
\hline & Merops hirundineus & 46,7 & 0,001 \\
\hline & Permis apivorus & 40 & 0,0022 \\
\hline & Ploceus melanocephalus & 53,3 & 0,0002 \\
\hline & Tutur afer & 53,3 & 0,0002 \\
\hline & Vanellus spinosus & 66,7 & 0,0002 \\
\hline & Vidua chalybeata & 40 & 0,0026 \\
\hline & Ardea melanocephala & 32,8 & 0,0362 \\
\hline & Lybius dubius & 44,4 & 0,0038 \\
\hline & Musophaga violacea & 30,5 & 0,086 \\
\hline & Numida meleagris & 27,2 & 0,1188 \\
\hline & Podica senegalensis & 21,8 & 0,2811 \\
\hline & Ptilopachus petrosus & 36 & 0,0302 \\
\hline & Tockus nasitus & 23,8 & 0,0912 \\
\hline & Plastysteria cyanea & 24 & 0,1526 \\
\hline \multirow[t]{31}{*}{ Soudanienne } & Amadina fasciata & 73,3 & 0,0002 \\
\hline & Anaplectes rubriceps & 40 & 0,0016 \\
\hline & Anastomus lamelligerus & 66,7 & 0,0002 \\
\hline & Anhinga rufa & 80 & 0,0002 \\
\hline & Anthoscopus parvulus & 46,7 & 0,001 \\
\hline & Ciconia abdimii & 33,3 & 0,006 \\
\hline & Ciconia episcopus & 60 & 0,0002 \\
\hline & Ciconia nigra & 46,7 & 0,001 \\
\hline & Cinnyris pulchellus & 86,7 & 0,0002 \\
\hline & Circus pygargus & 86,7 & 0,0002 \\
\hline & Cossypha albicapilla & 66,7 & 0,0002 \\
\hline & Criniger barbatus & 40 & 0,002 \\
\hline & Elminia longicauda & 73,3 & 0,0002 \\
\hline & Ephippirrhynchus senegalensis & 66,7 & 0,0004 \\
\hline & Estrilida caerulescens & 46,7 & 0,0012 \\
\hline & Estrilida tryglodytes & 46,7 & 0,0012 \\
\hline & Euodice cantans & 60 & 0,0002 \\
\hline & Laniarus barbarus & 33,3 & 0,0066 \\
\hline & Merops bulocki & 53,3 & 0,0004 \\
\hline & Melaenormis edoloides & 53,3 & 0,0002 \\
\hline & Mycteria ibis & 40 & 0,0018 \\
\hline & Oriolus auratus & 80 & 0,0002 \\
\hline & Plegadis falcinellus & 33,3 & 0,0084 \\
\hline & Ploceus vitellinus & 46,7 & 0,0002 \\
\hline & Pluvianus aegyptius & 40 & 0,0022 \\
\hline & Rhinoptilus chalcopterus & 46,7 & 0,001 \\
\hline & Streptopelia decipens & 40 & 0,0014 \\
\hline & Vanellus tectus & 73,3 & 0,0002 \\
\hline & Oxylophus Jacobinus & 24 & 0,1516 \\
\hline & Oxylophus levaillantii & 56,3 & 0,0006 \\
\hline & Heiliacetus vocifer & 32,8 & 0,0436 \\
\hline
\end{tabular}

VI : Valeur Indicatrice et P- Value : la valeur de la probabilité. 

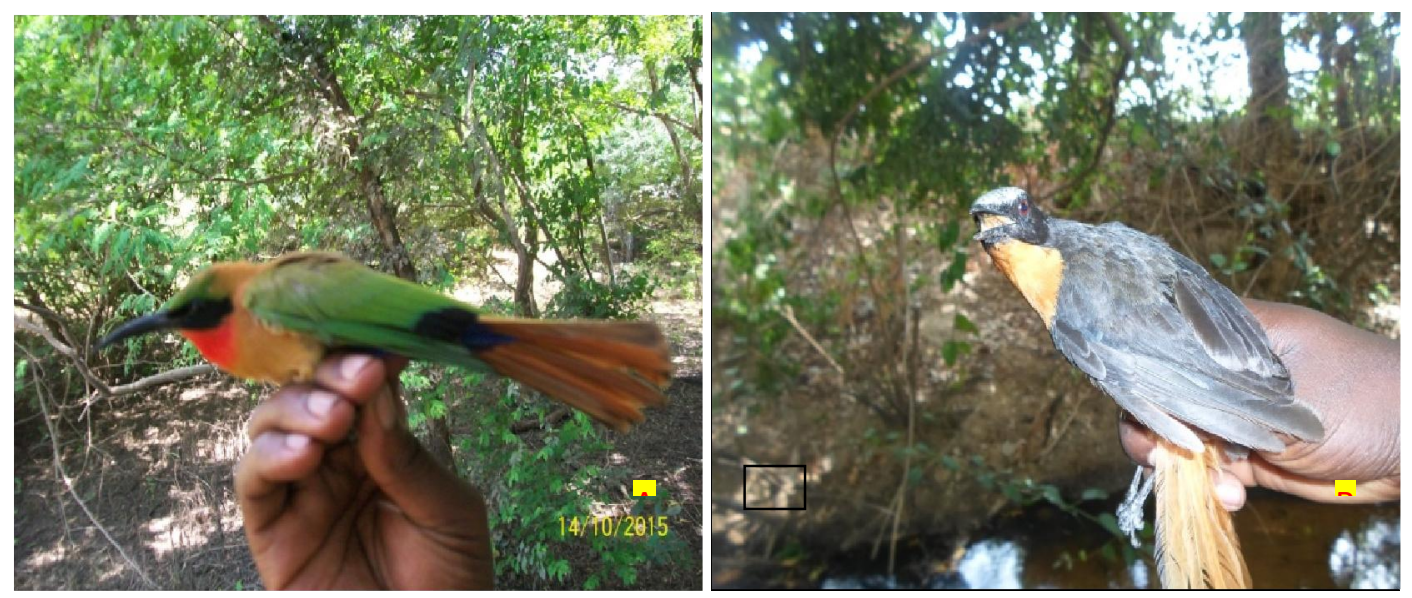

Photo 1: A : Merops bulocki, galerie forestière Alibori dans la zone soudanienne; B Cossypha albicapilla, galerie forestière Sota dans la zone soudanienne.
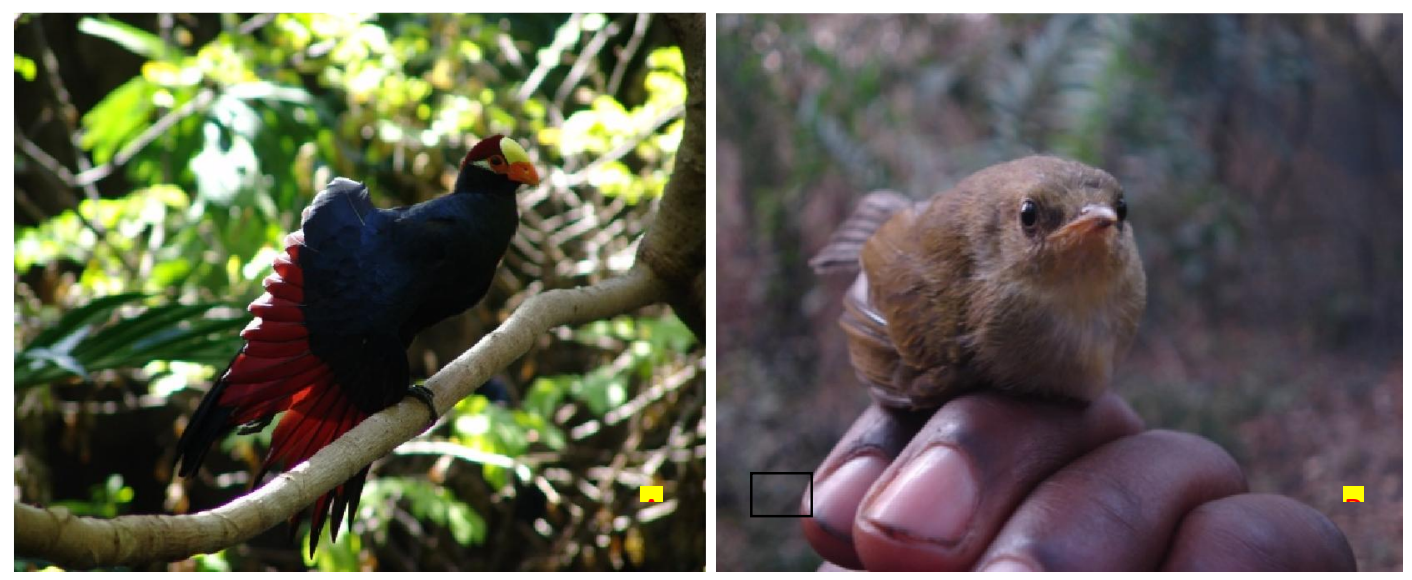

Photo 2 : C : Musophaga violacea, galerie forestière Ouémé dans la zone soudano - guinéenne, D : Apalis flavida, galerie forestière Okpara dans la zone soudano-guinéenne.

\section{DISCUSSION}

Espèces indicatrices des communautés d'oiseaux suivant les zones biogéographiques

La diversité élevée des oiseaux indicateurs des galeries forestières témoigne l'intérêt de cet écosystème pour la conservation de la diversité biologique avienne. Les galeries forestières à l'échelle du Bénin hébergent 140 espèces d'oiseaux dont
89 espèces sont indicatrices cet écosystème donnant respectivement une proportion de $26,62 \%$ et $16,92 \%$ de toute la composition avifaunistique (526 espèces d'oiseaux) du Bénin (Lougbegnon et Libois, 2011). Cette valeur est relativement faible par rapport à celle obtenue par Ahon et al. (2012) dans la forêt des marais de Tanoé - Ehy au Sud - Est de la Côte d'Ivoire. Ces résultats peuvent se justifier par le simple fait que son milieu 
d'étude a pris en compte plusieurs types d'écosystèmes à savoir: la lagune Ehy, le fleuve Tanoé, la forêt dégradée et la forêt dense. De plus, ces observations étaient diurnes et nocturne. Dans le contexte de cette recherche, nous avons pris exclusivement les galeries forestières et la période d'observation est diurne. Compris comme tel, dans notre liste, il n'y a que des espèces d'oiseaux diurnes. Sur le plan local quelques travaux existent et ont rapport d'une part sur les oiseaux forestiers du Sud Bénin et d'autres part sur les oiseaux gibiers des zones humides de la même partie. Les travaux de Lougbégnon et al. (2010) ont signalé la présence de 118 espèces d'oiseaux dans les écosystèmes forestiers du Sud du Bénin y compris les forêts galeries. Dans sa liste, l'auteur a signalé la présence de 47 espèces des zones humides. En comparaison avec les résultats de cette recherche, ces espèces ont été effectivement contactées dans les galeries forestières. De même, Lougbégnon (2015) signale également la présence de six espèces d'oiseaux gibiers les plus chassés par les populations au Sud du Bénin. Ces espèces sont entre autres Dendrocygna viduata, Egretta alba, Egretta ardesiaca, Porphyrio Porphyrio, Porphyrio alleni et Guttera pucherani et qui font parties des résultats obtenus dans cette recherche. Au nord du Bénin, les études de Yabi et al. (2015) ont signalé la présence de 53 espèces d'oiseaux dans les galeries forestières de la forêt classée des Monts Kouffé.

Les communautés d'oiseaux des galeries forestières de la zone guinnéenne sont particulières et spectaculaires comme l'indiquent les espèces indicatrices. Par exemple Batis poentis, Bleda canicapillus, Chlorocichla Simplex, Cyanomitra olivaceaus, Illadopsis fulvescens, Indicator maculatus, Lybius bidentatus, Merops pusillus, Nicator chloris, Pachycoccyx audeberti, Thescelocichla leucopleura, Macronyx croceus, Nigrita bicolor, Ploceus nigricollis, Terpsiphone rufiventer ont été décrits dans la littérature comme caractéristiques des galeries forestières situées dans la zone guinnéenne (Barow et Deme, 2001 ; Lougbégnon et al., 2009). Ces différentes espèces ont été contactées dans la partie Sud de la Côte d'Ivoire par les auteurs Yaokokore-Beibro et al. (2010) et Ahon et al. (2012).

Dans la zone Soudanienne, plus précisément dans le parc et les forêts classées, l'analyse des espèces indicatrices a révélé que les oiseaux Ardeola raloides et Vidua Amadina fasciata, Anaplectes rubriceps, Anastomus lamelligerus, Anhinga rufa, Anthoscopus parvulus, Ciconia abdimii, Ciconia episcopus, Ciconia nigra, Cinnyris pulchellus, Circus pygargus, Cossypha albicapilla, Elminia longicauda, Ephippirrhynchus senegalensis, Estrilida caerulescens, Estrilida tryglodytes, Euodice cantans, Laniarus barbarus, Merops bulocki, Merops nubicus, Melaenormis edoloides, Mycteria ibis, Oriolus auratus, Plegadis falcinellus, Ploceus vitellinus, Pluvianus aegyptius, Rhinoptilus chalcopterus, Podica senegalensis, Ptilopachus petrosus, Tockus nasitus sont des espèces typiquement indicatrices de la zone soudanienne. Bien la distribution de certaines espèces fait incursion dans la zone soudano-guinéenne, ces espèces sont souvent caractéristiques des galeries forestières à fort recouvrement de la végétation et par ricochet affectionnent les milieux à moindre intensité d'activité anthropique (Hassan et al., 2013). Les espèces 
indicatrices dans la zone soudano - guinéenne sont constituées des oiseaux Anthreptes longuemarei, Apalis flavida, Campephaga phoenicea, Glaucydium perlatum, Gypohierax angolensis, Lagonosticta larvata, Merops hirundineus, Permis apivorus, Ploceus melanocephalus, Tockus fasciatus, Vanellus spinosus, Vidua chalybeata, Ardea melanocephala, Lybius dubius, Musophaga violacea, Numida meleagris caractéristiques des galeries forestières anthropisées et des galeries forestières conservées à l'intérieure des forêts classée dans la zone soudanoguinnéenne. Certaines de ces espèces ont été confirmées par les études de Konan et Yaokokore-Beibro (2015) dans la région de Yamoussokro au centre de la Côte d'Ivoire. On peut postuler que les espèces d'oiseaux indicatrices se déplacent le long d'un gradient climatique en fonction du niveau de perturbation en présence dans les galeries forestières.

\section{Conclusion}

La présente recherche a permis de sélectionner les espèces d'oiseaux indicatrices des galeries forestières à travers les trois zones climatiques en présence au Bénin. On peut retenir que des 140 espèces d'oiseaux recensées, seules 89 espèces se sont révélées indicatrices des galeries forestières à l'échelle du Bénin. Les espèces d'oiseaux indicatrices des galeries forestières sont beaucoup plus majoritaires dans la zone soudanienne, suivie de la zone guinéenne et de la zone soudano guinéenne. Ces résultats témoignent du rôle cardinal que jouent les galeries forestières dans la conservation de la diversité biologique avienne. De ce point de vue, on comprend donc la nécessité d'intégrer les galeries forestières dans le réseau des aires protégées au Bénin. Compris comme tel, il serait bien d'asseoir une bonne stratégie de conservation des galeries forestières en se basant sur les indicateurs de santé environnementale que sont les oiseaux.

\section{CONTRIBUTIONS DES AUTEURS}

FBY a réalisé les travaux, collecté les données et a rédigé le manuscrit. TOL a corrigé le protocole. Il a également vérifié les fiches de collecte des données et a supervisé le traitement et l'analyse des résultats. JTCC a situé le contexte et défini l'objectif de cet article. Il a suivi et apporté ces critiques pendant les différentes étapes de la rédaction. Il a aussi approuvé la méthode adoptée et participé à la correction du manuscrit.

\section{CONFLIT D'INTERETS}

Les auteurs déclarent qu'ils n'ont aucun conflit d'intérêt.

\section{REFERENCES}

Adomou AC, Akoègninou A, Sinsin B, De Foucault B, Van der Maesen LJG. 2006. Biogeographical analysis of the vegetation in Benin. Acta Bot. Gallica, 154(2): 221-233.

Ahon DB, Egnankou WM, Kouadio KR, Kouame OML. 2012. Inventaires préliminaires des oiseaux de la forêt des marais Tanoé-Ehy en Côte d'Ivoire. International Journal of Biological and Chemical Sciences, 6(6): 4031-4045.

Akoègninou $\mathrm{A}$, van der Burg $\mathrm{WJ}$, van der Maesen LJG. 2006. Flore Analytique du Bénin. Leinden, Backhuys: 1034 P.

Bennum LA. 2000. Monitoring bird population in Africa: an overview. Ostrich, 71: 214- 215. 
Borrow N, Demey R. 2001. Birds of Western Africa. Christopher Helm: London; 832 p.

Borrow N, Demey R. 2003. Birds of Western Africa. Christopher Helm: London; $511 \mathrm{p}$.

Büchs W. 2003. Biotic indicators for biodiversity and sustainable agriculture-introduction and background. Agriculture, Ecosystems \& Environment, 98: 1-16.

Carignan V, Villard M. 2002. Selecting indicator species to monitor ecological integrty: A review. Environmental Monitoring, $78: 45-61$.

CENATEL, 2002. Rapport final de base de données géoréférencées sur l'utilisation agricole des terres au Bénin, Rapport d'étude, Bénin, 20 p.

Chessel, Thioulouse, 2001. Analyse de données spatialisées. In Biométrie et Biologie Evolutive, Cours de Biostatistique. Université de Lyon I; 30 p.

Delahaye L. 2006. Sélection et modélisation de l'habitat d'oiseaux en chênaies et hêtraies ardennaises: étude de l'impact de la composition et de la structure forestière. Thèse de doctorat, Univ. de Gembloux, 401 p.

Dufrêne M. 2001. Indval or how to identify indicator species of a sample typology? http//mrw.walonie.be/dgrne/sibw/outils/i ndval/home.html ed, vol 2004, MRW.

Dufrêne M, Legendre P. 1997. Species assemblages and indicator species: the need for a flexible asymetrical approach. Ecological Monographs, 67: 345-366.

Hassan SN, Salum A, Rija AA, Modest R, Kideghesho JR, Malata PF. 2013. Human-induced Disturbances Influence on Bird Communities of Coastal Forests in Eastern Tanzania. British Journal of Applied Science \& Technology, 3(1): 4864.

Khaffou M, Chalaoui A, Samih M. 2013. Les habitats utilisés par le Tadorne casarca (Tadorna ferruginea) dans la zone humide d'Aguelmam Sidi Ali - Site Ramsar - Moyen Atlas - Maroc. International Journal of Biological and Chemical Sciences, 7(2): 598-606.

Konan EM, Yaokokoré-Beibro KH. 2015 Variation temporelle du peuplement aviaire des écosystèmes lacustres de la ville de Yamoussoukro, centre de la Côte d'Ivoire. International Journal of Biological and Chemical Sciences, 9(6): 2566-2581.

Lougbégnon OT, Codjia JTC, Libois MR. 2009. Sélection d'espèces indicatrices d'oiseaux des habitats forestiers et de substitution du Sud du Bénin, implication pour une gestion des milieux naturels. Climat et Développement, 7: 85-97.

Lougbégnon TO. 2015. Ecologie et connaissance ethnozoologique de quelques espèces d'oiseaux gibiers menaces des écosystèmes du sud du Bénin. Thèse de doctorat, Université de Liège, Belgique, $146 \mathrm{p}$.

Lougbegnon TO. 2008. Biodiversité, écologie et conformation morphologique des oiseaux forestiers naturels du sud du Bénin. Thèse de doctorat unique, Université d'Abomey - Calavi, Bénin, $139 \mathrm{p}$.

Lougbégnon OT, Libois RM. 2011. Protection de la nature en Afrique de l'Ouest : une liste rouge pour le Bénin, Nature conservation In West Africa : Red List 
for Benin. Chap. 19. Oiseaux, Birds. P. Neuenschwander BS, Goergen G (eds). International Institute of Tropical Agriculture : Ibadan, Nigeria; 204-228

Lougbenon TO, Codjia JC, Libois RM. 2010. Distribution de l'avifaune des milieux forestiers de substitution (plantation et jachères) au Sud du Bénin en relation avec les facteurs de l'habitat. International Journal of Biological and Chemical Sciences, 4(4): 1191-1216.

Nahmani J, Lavelle P, Rossi JP. 2006. Does changing the taxonomical resolution alter the value of soil macroinvertebrates as bioindicators of metal pollution? Soil Biology \& Biochemistry, 38: 385-396.

PNUD-MEPN. 2009. Quatrième rapport national du Bénin sur la convention des nations unies sur la diversité biologique. Cotonou, Bénin, 130p.

Rija AA, Said A, Mwamende KA, Hassan SN, Madoffe SS. 2014. Urban sprawl and species movement may decimate natural plant diversity in an Afro-tropical city.
Biodiversity \& Conservation, 23: $963-$ 978.

Seymour CL, Simmons RE, Joseph GS, Slingsby JA. 2015. On Bird Functional Diversity: Species Richness and Functional Differentiation Show Contrasting Responses to Rainfall and Vegetation Structure in an Arid Landscape. Ecosyst., 18: 971-984.

Yabi BF, Lougbégnon OT, Houessou GH, Codjia JTC. 2015. Effets de l'anthropisation sur la diversité de l'avifaune des galeries forestières de la forêt classée des Monts Kouffé au Bénin. Afrique Science, 11(6): 334 - 348, http://www.afriquescience.info.

Yaokokoré-Béibro HK, N'guessan MA, Odoukpé KSG, Zouzou EJ, N'douba V, Kouassi PK. 2010. Premières données sur les oiseaux de la zone humide d'importance internationale de GrandBassam (Côte d'Ivoire). International Journal of Biological and Chemical Sciences, 4(6): 2169-2180. 